\title{
Crossover to Quantized Thermal Conductance in Nanotubes and Nanowires
}

\author{
Kohei Yamamoto $^{1}$, Hiroyuki Ishii ${ }^{1}$, Nobuhiko Kobayashi ${ }^{{ }^{*}}$, Kenji Hirose ${ }^{2}$ \\ ${ }^{1}$ Institute of Applied Physics and Tsukuba Research Center for Interdisciplinary Materials Science, University of Tsukuba, Tsukuba, \\ Japan; ${ }^{2}$ Green Innovation Research Laboratory, NEC Corporation, Tsukuba, Japan. \\ Email: ${ }^{*}$ nkoba@bk.tsukuba.ac.jp
}

Received February $28^{\text {th }}, 2013$; revised March $28^{\text {th }}, 2013$; accepted April $18^{\text {th }}, 2013$

Copyright (C) 2013 Kohei Yamamoto et al. This is an open access article distributed under the Creative Commons Attribution License, which permits unrestricted use, distribution, and reproduction in any medium, provided the original work is properly cited.

\begin{abstract}
Using the non-equilibrium Green's function techniques with interatomic potentials, we study the temperature dependence and the crossover of thermal conductance from the usual behavior proportional to the cross-sectional area at room temperature to the universal quantized behavior at low temperature for carbon nanotubes, silicon nanowires, and diamond nanowires. We find that this crossover of thermal conductance occurs smoothly for the quasi-one-dimensional materials and its universal behavior is well reproduced by the simplified model characterized by two parameters.
\end{abstract}

Keywords: Carbon Nanotube; Nanowire; Thermal Transport; Quantum Conductance; Non-Equilibrium Green's Function

\section{Introduction}

Recently, phonon thermal transport properties of quasione-dimensional materials, such as carbon nanotubes (CNTs) and silicon nanowires (SiNWs), have attracted much attention in the fields of nanometer-scale electron devices and thermoelectric devices. The high thermal conductance opens the way to reduce the heating problems in nanometer-scale electron devices, while low thermal conductance leads to high efficiency of thermoelectric devices [1]. Consequently, it is very important to know how the quasi-one-dimensional materials conduct heat. It is predicted that thermal conductance is quantized as $G(T) \propto g_{0} \equiv\left(\pi^{2} / 3\right)\left(k_{B}^{2} T / h\right)$ at low temperature and this quantization dose not depend on kinds of materials and carriers [2]. Indeed, this $g_{0}$ is experimentally measured for phonons [3], electrons [4], and even photons [5]. However, it is known that this quantization breaks at high temperature. Moreover, in macroscopic, the conductance should be proportional to the cross-sectional area of wires [6]. We investigate this region between quantized and macroscopic thermal conduction. For such studies, including quantized thermal conductance, we need the computational approach taking the quantum effects explicitly into account. The Landauer formula [7] or the non-equilibrium Green's func-

${ }^{*}$ Corresponding author. tion (NEGF) technique [8] has been widely studied. In addition to electron transport $[9,10]$, these methods have recently been applied to the thermal transport [11] and the thermal conductance is calculated for SiNWs [12] and CNTs [13]. Recently using the NEGF technique, the quantized thermal conductance has been analyzed for SiNWs [14]. According to the calculation, thermal conductance is quantized not proportional to diameter at all at low temperature and has a diameter dependence gradually as the temperature increases. Over the temperature $100 \mathrm{~K}$, the thermal conductance becomes proportional to cross-sectional area of nanowires.

Here, we report the thermal conductance of the quasione-dimensional systems, in particular, diameter dependence for materials such as CNTs, SiNWs, and diamond nanowires (DNWs) at various temperatures. Using a simplified model based on the assumption for the phonon dispersion relations, we first show the general behaviors of the crossover to quantized thermal conductance from the usual behavior, proportional to the cross-sectional area, to the unusual "quantum-type", not dependent on the cross-sectional area or the diameter with decreasing temperature. Especially, we consider the following model. Since the quasi-one-dimensional materials have four acoustic phonon modes; one longitudinal mode, two flexural modes, and one torsional mode, we propose the model that these modes are well separated in the phonon 
dispersion relation from the other optical modes, the number of which is proportional to the number of atoms in cross-section of materials. We can obtain the universal thermal conductance behavior with only two parameters to describe the present simplified model. Then, we present the elaborate atomistic calculations for the thermal conductance of various realistic systems using the NEGF technique. We explain the difference of the temperature dependence of thermal conductance by comparing with those for the simplified model and clarify the important two parameters to account for the crossover to the quantized thermal conductance.

\section{Simplified Model}

First, we introduce a simplified model to discuss the temperature dependence of thermal conductance of quasi-one-dimensional systems. In particular, we try to analyze qualitatively the crossover to quantized thermal conductance from the usual conductance, which is proportional to its cross-sectional area. Here we note that in the Debye approximation where the phonon dispersion relation for the quasi-one-dimensional model composed of mono-atomic chain is approximated by the simple linear dispersion as shown in Figure 1(a), there apparently shows no crossover of thermal conductance since the transmission coefficient exists only for that phonon
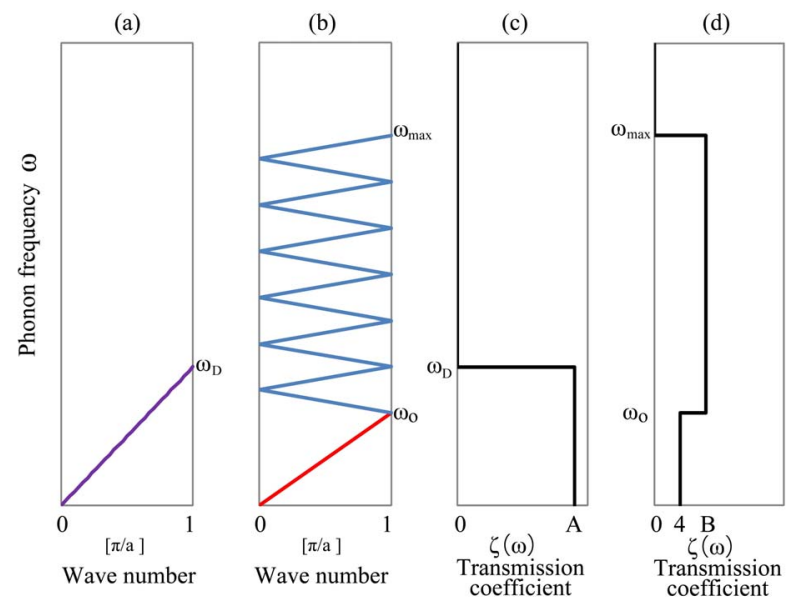

Figure 1. (a) Phonon dispersion relation of a quasi-onedimensional mono-atomic model for the acoustic mode in the debye approximation; (b) Schematic picture of phonon dispersion relation in the present simplified model, which is composed of four acoustic phonon modes for $\omega<\omega_{0}$ and the other uniform phonon modes for $\omega_{0}<\omega<\omega_{\max }$; (c) Typical transmission coefficient $\zeta(\omega)$ as a function of phonon frequency $\omega$ for the one-dimensional monoatomic model in (a); (d) Transmission coefficient $\zeta(\omega)$ in the simplified model. $B(\equiv \beta N)$ is the number of phonon modes at $\omega$, where we assume $B$ is proportional to the number of atoms $N$ in the cross-sectional area. mode (Figure 1(c)).

Instead, we consider a system for the phonon thermal transport which is described by two dimensionless parameters $\Omega$ and $\beta$. Here $\Omega$ indicates the ratio of $\omega_{0} / \omega_{\max }$, where $\omega_{\max }$ is the maximum phonon frequency for all the phonon modes and $\omega_{0}$ is the phonon frequency under which only four acoustic phonon modes exist. It should be noted that the torsional mode appears in the quasi-one-dimensional systems in addition to the one longitudinal mode present typically in one-dimensional system as in Figure 1(a) and two flexural modes present for bulk system. Important point is that we consider these four modes are well separated from the other phonon modes which exist in the higher energies and play the important role for the quantized thermal conductance at low temperature. As the temperature increases, various phonon modes are excited beyond these acoustic modes. In general, the degrees of the freedom increase linearly with the number of the atoms and the number of the phonon bands increases correspondingly.

In the region of frequencies between $\omega_{0}$ and $\omega_{\max }$, we assume for simplicity that the number of the phonon modes becomes uniform and is proportional to the number of atoms $N$ in the cross-sectional area. Since the transmission coefficient $\zeta(\omega)$ corresponds to the number of phonon modes for ballistic transport, we obtain $\zeta=\beta N$, where we introduce the parameter $\beta$ as the proportional constant. As an example of the simplified model, we show a schematic phonon dispersion relation and the corresponding transmission function $\zeta(\omega)$ in Figures 1(b) and (d), respectively.

The thermal current $J_{t h}$ for the system is expressed in the Landauer's type as follows, [2]

$$
J_{t h}=\int_{0}^{\infty} \frac{d \omega}{2 \pi} \hbar \omega\left[n_{B E}\left(\omega, T_{L}\right)-n_{B E}\left(\omega, T_{R}\right)\right] \zeta(\omega),
$$

where $n_{B E}\left(\omega, T_{L(R)}\right)$ is the Bose-Einstein distribution function of equilibrium phonons with an energy of $\hbar \omega$ in the left (right) lead at temperature $T_{L(R)}$. For adiabatic contact between the wire and the leads, $\zeta(\omega)$ is expressed in the present model as follows,

$$
\zeta(\omega)=\left\{\begin{array}{ll}
4 & \left(\omega \leq \omega_{0}\right) \\
\beta N & \left(\omega_{0} \leq \omega \leq \omega_{\max }\right) \\
0 & \left(\omega_{\max } \leq \omega\right)
\end{array} .\right.
$$

In the limit of small temperature difference between the left and right leads, the temperature dependence of thermal conductance $G(T)$ is given by

$$
\begin{aligned}
G(T)= & \frac{\mathrm{d} J_{t h}}{\mathrm{~d} T} \\
& =\frac{k_{B}}{2 \pi} \times 4 \int_{0}^{\omega_{0}} \mathrm{~d} \omega \frac{\hbar \omega}{k_{B}} \frac{\partial n_{B E}(T)}{\partial T} \\
& +\frac{k_{B}}{2 \pi} \beta N \int_{0}^{\omega_{\max }} \mathrm{d} \omega \frac{\hbar \omega}{k_{B}} \frac{\partial n_{B E}(T)}{\partial T}
\end{aligned}
$$


The thermal conductance $G(T)$ shows explicitly the size dependence for $N$ in addition to the temperature dependence. At low temperature the first term dominates the thermal conductance and $G(T)$ shows the quantized thermal conductance, without any dependence of $N$, a universal features as $G(T)=4 g_{0}$. As the temperature increases, the contribution from the second term having the size dependence plays a dominant role. Since this term is proportional to $N$, that is, its cross-sectional area, it represents the usual thermal conductance. With use of these expressions, we calculate the temperature dependence of the thermal conductance to discuss the crossover region between the quantized conductance and the usual conductance.

In order to express the characteristic feature on the temperature dependence of thermal conductance explicitly, let us extrapolate the thermal conductance by the power-law as $G(T) \propto N^{n}$. This enables us to discuss the crossover from $n=0(T \rightarrow 0)$ to $n=1(T \rightarrow \infty)$ for various temperatures. Figure 2 shows the exponent $n$ as a function of the temperature. The temperature is measured in units of $\hbar \omega_{\max } / k_{B}$. The horizontal axis cor-
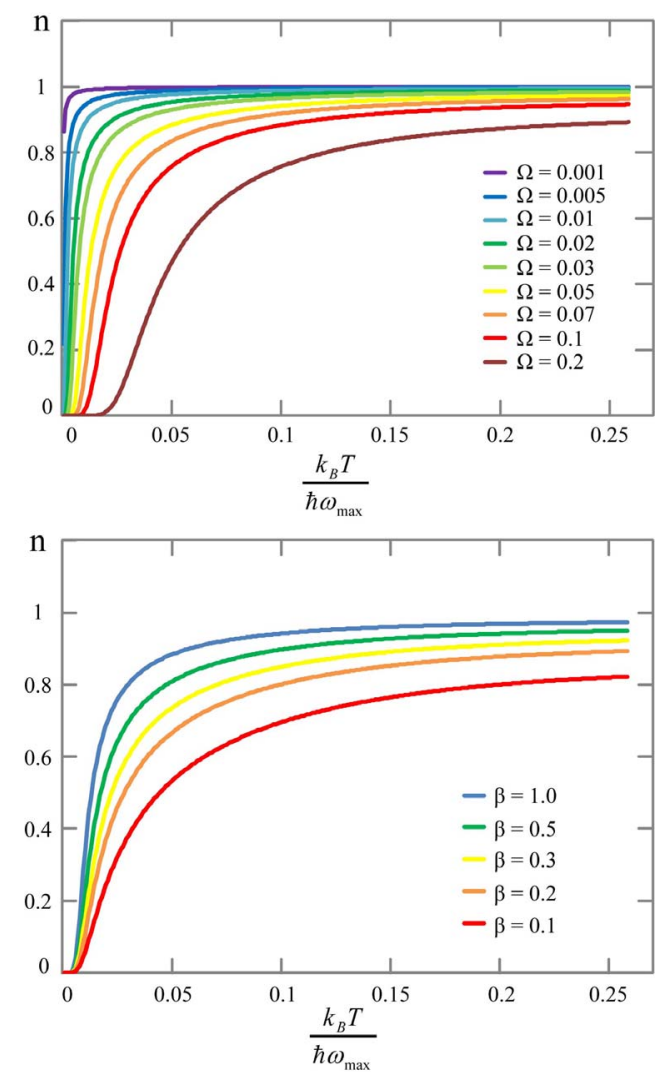

Figure 2. (Top) Exponent $n$ of thermal conductance $G(T) \propto N^{n}$ as a function of temperature $k_{B} T / \hbar \omega_{\max }$ for various parameters of $\Omega=\omega_{0} / \omega_{\max }$ with $\beta=1$; (Bottom) Exponent $n$ as a function of $k_{B} T / \hbar \omega_{\max }$ for various parameters of $\beta$ with $\Omega=\mathbf{0 . 0 5}$. responds to the temperatures from 0 to $300 \mathrm{~K}$ for the maximum phonon energy of typical parameter of $\hbar \omega_{\max }$ of $100 \mathrm{meV}$. We note that this parameter value would be different from material to material in the realistic systems. It is observed that smooth transition curves from the quantized conductance regime $(n=0)$ to the usual thermal conductance regime $(n=1)$ are obtained. The upper panel shows the exponent $n$ for various $\Omega$ with $\beta=1$. The onset temperature is determined by $\Omega$ because only four phonon modes exist under the frequency $\omega_{0}$ leading to the quantized conductance. With an increase of temperature, the second term dependent on $N$ in Equation (3) enhances and $n$ approaches to one. The lower panel shows the exponent for various $\beta$ with $\Omega=0.05$. As the parameter $\beta$ becomes smaller or $\Omega$ becomes larger, the asymptotic behavior of the exponent $n$ to approach the value of $n=1$ becomes slower. This is because the first term which is independent of $N$ in Equation (3) becomes not negligible in this case.

These calculations suggest that qualitative general behaviors might be elucidated using a simplified model based on the assumption of simple phonon dispersion relation. We note that these characteristic features are represented by only two critical parameters, $\Omega$ and $\beta$. Realistic materials have more complex phonon dispersions. In the next section, we perform more elaborate atomistic calculations for the phonon dispersion and the thermal transport to investigate the details of crossover to the quantized thermal conductance and study the validity of the present simplified model.

\section{Realistic Atomistic Calculations}

Next, we consider the temperature dependence of thermal conductances of realistic quasi-one-dimensional materials. As typical examples, we take SiNWs, DNWs, and CNTs, and analyze the phonon transport properties from an atomistic viewpoint. The Hamiltonian $H$ for the present phonon transport is expressed as follows:

$$
H=\sum_{\substack{i \in S y s \\ \alpha=x, y, z}} \frac{1}{2 M_{i}} p_{i \alpha}^{2}(t)+\frac{1}{2} \sum_{\substack{i, j \in s y s \\ \alpha, \beta=x, y, z}} u_{i \alpha}(t) K_{i \alpha, j \beta} u_{j \beta}(t) .
$$

Here $M_{i}$ is a mass of $i$-th atom and $u_{i \alpha}(t)$ is an operator for displacement of $i$-th atom along $\alpha$ direction from equilibrium position, respectively. We split the total Hamiltonian into four pieces:

$$
H=H_{L}+H_{S}+H_{R}+H_{\text {int }},
$$

where $H_{L(R)}$ is the Hamiltonian for the left (right) lead, $H_{S}$ is for the scattering region, and $H_{\text {int }}$ is for the interaction between the scattering region and the left (right) lead. Using the NEGF technique [11], the thermal current $J_{t h}$ and the thermal conductance $G(T)$ from the left lead to the scattering region can be expressed in the similar form as Equations (1) and (3) such as 


$$
\begin{aligned}
J_{t h} & =-\left\langle\dot{H}_{L}\right\rangle \\
& =\int_{0}^{\infty} \frac{\mathrm{d} \omega}{2 \pi} \hbar \omega\left[n_{B E}\left(\omega, T_{L}\right)-n_{B E}\left(\omega, T_{R}\right)\right] \zeta(\omega) \\
& G(T)=\frac{k_{B}}{2 \pi} \int_{0}^{\infty} \mathrm{d} \omega \frac{\hbar \omega}{k_{B}} \frac{\partial n_{B E}}{\partial T} \zeta(\omega) .
\end{aligned}
$$

Here the bracket $\langle\cdots\rangle$ denotes the non-equilibrium statistical average of the physical observable and $\zeta(\omega)$ is the transmission coefficient for the phonon transport through the scattering region given by

$$
\zeta(\omega)=\operatorname{Tr}\left[\Gamma_{L}(\omega) G^{r}(\omega) \Gamma_{R}(\omega) G^{a}(\omega)\right] .
$$

Here, $G^{r / a}(\omega)$ is the retarded/advanced Green's function for the scattering region and $\Gamma_{L / R}(\omega)$ is the coupling constant. For the ideal ballistic limit without any scatterings, $\zeta(\omega)$ is equal to the number of phonon subbands at frequency $\omega$.

The retarded/advanced Green's function for the scattering region is given by

$$
G^{r / a}(\omega)=\left[\omega^{2} M-K-\Sigma_{L}^{r / a}-\Sigma_{R}^{r / a}\right]^{-1},
$$

where $M$ is the diagonal matrix whose element is a mass of a silicon or a carbon atom and $\sum_{L / R}^{r / a}(\omega)$ is the retarded/advanced self-energy due to the coupling to the left/right semi-infinite lead with the scattering region, which is obtained independently from the atomistic structure of the lead. We use a quick iterative scheme with the surface Green's function technique [15] to calculate the self-energy for complex atomic structures of nanowires. The coupling constant $\Gamma_{L / R}(\omega)$ in Equation (6) is then obtained from

$$
\Gamma_{L / R}(\omega)=i\left[\Sigma_{L / R}^{r}(\omega)-\Sigma_{L / R}^{a}(\omega)\right] .
$$

The dynamical matrix $K$ which is contained in the total Hamiltonian is constructed from the force constants between the atoms. The matrix elements of $K_{i \alpha, j \beta}$ are calculated by finite difference of the force $F_{i \alpha}$ with respect to $r_{j \beta}$ as

$$
K_{i \alpha, j \beta}=\frac{\partial^{2} E}{\partial r_{i \alpha} \partial r_{j \beta}}=\frac{-\left[F_{i \alpha}\left(+\Delta R_{j \beta}\right)-F_{i \alpha}\left(-\Delta R_{j \beta}\right)\right]}{2 \Delta R_{j \beta}}
$$

The force $F_{i \alpha}$ is obtained from the derivative of $E$ with respect to $r_{i \alpha}$, where $E$ is the total energy of the system and $r_{i \alpha}$ is the atomic coordinate of the $i$-th atom along the $\alpha$ direction. Therefore $F_{i \alpha}\left(+\Delta R_{j \beta}\right)$ indicates the force of the $i$-th atom along the $\alpha$ direction generated by the $j$-th atom along the $\beta$ direction with a displacement of $+\Delta R$ from the pristine wire's equilibrium positions. Here $\Delta R$ is a displacement, for which we take $\Delta R=2 \times 10^{-4} \AA$ in the present work. As for the total energy formula $E$, we use the interatomic Tersoff potential [16] for silicon systems and Brenner potential [17] for carbon systems. These potentials describe the interatomic potentials with only elementary functions of atomic coordinates. The Tersoff (Brenner) potential describes total energy as follows:

$$
\begin{gathered}
E=\sum_{i} E_{i}=\frac{1}{2} \sum_{i \neq j} V_{i j} \\
V_{i j}=f_{c}\left(r_{i j}\right)\left[a_{i j} A e^{-\lambda_{1} r_{i j}}+b_{i j} B e^{-\lambda_{2} r_{i j}}\right] .
\end{gathered}
$$

Here, $A, B, \lambda_{1}$ and $\lambda_{2}$ are constants, $r_{i j}$ is a distance between the $i$-th and $j$-th atoms and $a_{i j}$ and $b_{i j}$ are determined by only atomic coordinates.

Figures 3(a)-(c) show the phonon dispersion relations

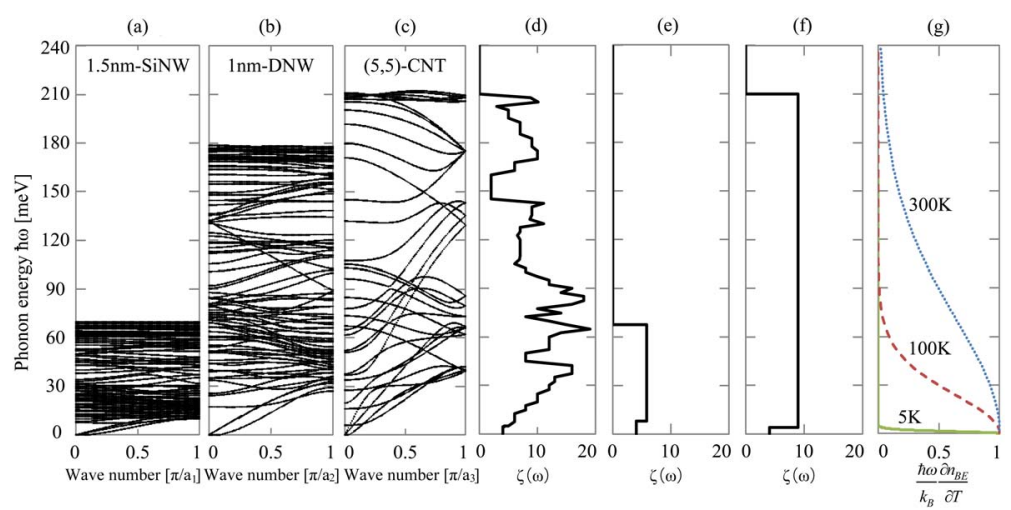

Figure 3. Phonon dispersion relations of (a) $\langle 100\rangle$-SiNW with $1.5 \mathrm{~nm}$ in diameter; (b) $\langle 100\rangle$-DNW with $1 \mathrm{~nm}$ in diameter; and (c) (5,5)-CNT. Here, $a_{1}=5.362 \AA, a_{2}=3.567 \AA$, and $a_{3}=2.49 \AA$ are unit vectors of the SiNW, DNW, and CNT, respectively; (d) Transmission function $\zeta(\omega)$ for the phonon dispersion relation of (5,5)-CNT; (e) $\zeta(\omega)$ corresponding to the simplified model for the $\langle 100\rangle$-SiNW; (f) $\zeta(\omega)$ corresponding to the simplified model for the (5,5)-CNT; (g) Differentials of the distribution functions $n_{B E}(\omega, T)$ with respect to $T$ for $5 \mathrm{~K}$ (green solid line), $100 \mathrm{~K}$ (red dashed line), and $300 \mathrm{~K}$ (blue dotted line). Note that the integral of its product with $\zeta(\omega)$ corresponds to $G(T)$. 
of a $\langle 100\rangle-S i N W$ with a diameter of $1.5 \mathrm{~nm}$, a $\langle 100\rangle-\mathrm{DNW}$ with a diameter of $1.0 \mathrm{~nm}$, and a $(5,5)-\mathrm{CNT}$ respectively. Since the SiNW and the DNW have the same atomic configurations with the same numbers of atoms in cross-sectional areas, these two phonon bands have similar structures with a difference of the phonon energy range, for which DNW has a larger value due to the stronger interatomic force acting between carbon atoms. On the other hand, the CNT has a cylindrical shape and each band of the CNT tends to have dispersions with a wide energy range, which induces large thermal conductance. Figures 3(d) and (g) show the transmission function $\zeta(\omega)$ for the CNT and the differentials of the distribution functions $n_{B E}(\omega, T)$ as a function of the phonon energy. The integral of these products corresponds to the thermal conductance $G(T)$.

At small phonon energy regime, we see that all quasione-dimensional structures have four acoustic modes, one longitudinal, two flexural, and one torsional modes, which do not depend on the detailed structures of nanowires. When only these four modes conduct heat, thermal conductance shows a universal feature for any material or diameter. This accounts for the quantized thermal conductance at low temperature regime [2]. As the temperature increases, various phonon modes begin to contribute to the thermal transport.

Here, we compare the behaviors of thermal conductance for SiNWs and CNTs, which have completely different atomic configurations as nanowires. The bottom panels of Figure 4 show the temperature dependences of
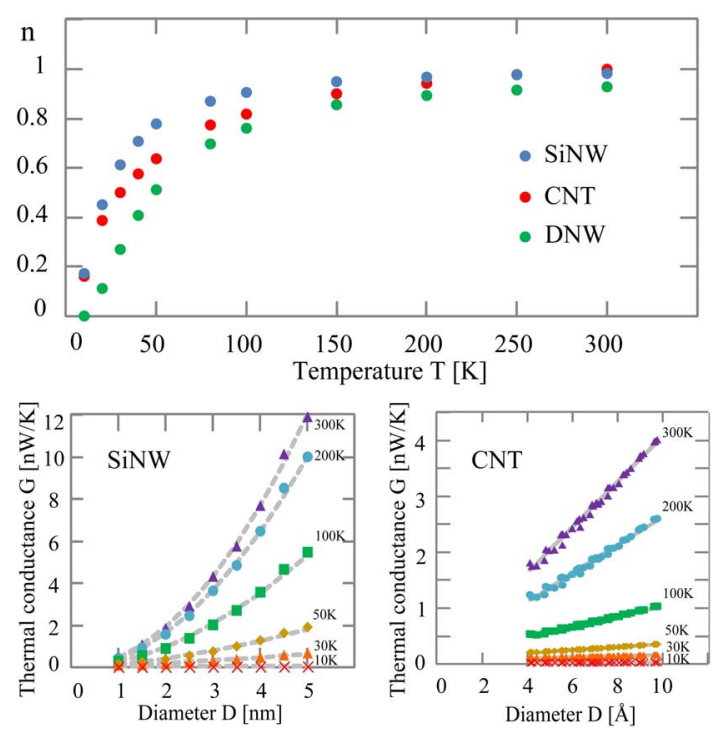

Figure 4. (Top) Exponent $n$ for several temperatures extrapolated from the thermal conductance as described by $G \propto N^{n}$, where $N$ is the number of atoms in the crosssection area. (Bottom) Thermal conductance as a function of the diameter $D$ for several temperatures in cases of the SiNW (left) and the CNT (right). the calculated thermal conductances as a function of the diameter $D$ for the SiNW (left) and the CNT (right). At $300 \mathrm{~K}$ the thermal conductances are proportional to the square of the diameter $D^{2}$ for SiNWs (left) and to the diameter $D$ for CNTs (right). Since the SiNWs have columnar shapes and the CNTs have cylindrical shapes, $N \propto D^{2}$ for the SiNWs and $N \propto D$ for the CNTs. This indicates that the thermal conductance is proportional to the cross-sectional area at high temperature. As the temperature decreases from $300 \mathrm{~K}$, the behaviors of thermal conductances to the diameter dependence are seen to change gradually. At enough low temperature, we see no dependence on a diameter $D$ for the thermal conductance $G(T)$ for both cases.

To analyze the temperature dependence of the thermal conductance, as we did in the previous section, we extrapolate the exponents $n$ for the thermal conductance $G(T) \propto N^{n}$ for the SiNWs, DNWs and CNTs, which are shown in the top panel of Figure 4. At $300 \mathrm{~K}$, the exponents of wires are nearly equal to 1 which correspond to usual thermal conductance and the exponents approach to 0 which correspond to "quantum-type" thermal conductance as the temperature decreases. We see that the crossover to $n=0$ from $n=1$ differs slightly according to the kinds of materials. The rapid decrease of the exponent $n$ from the saturation appears at the lowest temperature for SiNWs. This can be understood from the scaling of the temperature by $\hbar \omega_{\max } / k_{B}$ as shown in Figures 3(e) and (f), where $\omega_{\max }$ are 70 $\mathrm{meV}$ for SiNWs, $180 \mathrm{meV}$ for DNWs, and $210 \mathrm{meV}$ for CNTs. Among these materials the normalized temperature of SiNWs is the lowest, which is also related to the Debye temperature in the specific heat. To compare the behaviors of thermal conductance between DNWs and CNTs which have similar $\omega_{\max }$, we see that the onset temperature of CNTs is lower. This can be understood because $\Omega=\omega_{0} / \omega_{\max }$ is smaller for CNTs than DNWs. We note that another parameter of the proportional constant $\beta$ is determined so that $\zeta(\omega)$ in the simplified model has the same area as that in the realistic atomic system.

These data show that the simplified model as introduced in the previous section is effective to understand the behaviors of crossover to quantized thermal conductance, even if it is expressed with two parameters by the abbreviation of the precise transmission coefficients $\zeta(\omega)$ to the simplified ones as shown in Figures 3(e) and (f). Here we note that, in the present theoretical scheme, thermal conductance should not necessarily be proportional to the cross-sectional area in the high temperature limit, since the contribution to the transport from each phonon band depends on the width of the band dispersion, while the number of total phonon modes is proportional to the number of atoms in a unit cell. 
Phonon-phonon scattering effect is not taken into account here. Experimentally it has been observed that for nanowires less than $37 \mathrm{~nm}$ thick, the effect of anharmonicity on the thermal conductivity is not significant up to room temperature. Instead, the phonon boundary scattering due to surface roughness becomes important for the nanowires [18]. The effect is also discussed theoretically [12]. It is shown that anharmonicity is suppressed for nanowires shorter than tens of nanometers. We consider the clean wire case, where no edge or internal scatterings are present simply. However, note that such scatterings have the effects on the thermal conductance in the length direction $L$. We have checked the effects of the boundary scattering due to surface roughness in the SiNW on the exponent $n$. We remove 5\% atoms from the surface randomly with 10 different configurations and treat the results statistically. As a result, we find that the trend of the exponential behavior of the conductance from $n=1$ to 0 remains for the boundary scattering although the conductance reduces.

\section{Conclusion}

We have proposed a simplified model to discuss the crossover to universal quantized thermal conductance of the quasi-one-dimensional systems. From the calculations of the temperature dependence of thermal conductance for the realistic atomistic systems of CNTs, SiNWs, and DNWs, for which the crossover from the usual behavior at room temperature to the universal "quantum-type" behavior at low temperature is obtained by using the non-equilibrium Green's function techniques with interatomic potentials, we find that this crossover occurs smoothly for the quasi-one-dimensional materials and its universal behavior is well reproduced by the simplified model characterized by two parameters.

\section{Acknowledgements}

This work is supported in part by a Grant-in-Aid for Scientific Research from the Ministry of Education, Culture, Sports, Science and Technology of Japan.

\section{REFERENCES}

[1] A. I. Boukai, Y. Bunimovich, J. T. Kheli, J.-K. Yu, W. A. Goddard III and J. R. Heath, "Silicon Nanowires as Efficient Thermoelectric Materials," Nature, Vol. 451, 2008, pp. 168-171. doi:10.1038/nature06458

[2] L. G. C. Rego and G. Kirczenow, "Fractional Exclusion Statistics and the Universal Quantum of Thermal Conductance: A Unifying Approach," Physical Review B, Vol. 59, No. 20, 1999, pp. 13080-13086. doi:10.1103/PhysRevB.59.13080

[3] K. Schwab, E. A. Henriksen, J. M. Worlock and L. Roukes, "Measurement of the Quantum of Thermal Conduc- tance," Nature, Vol. 404, 2000, pp. 974-977. doi: $10.1038 / 35010065$

[4] O. Chiatti, J. T. Nicholls, Y. Y. Proskuryakov, N. Lumpkin, I. Farrer and D. A. Ritchie, "Quantum Thermal Conductance of Electrons in a One-Dimensional Wire," Physical Review Letters, Vol. 97, 2006, Article ID: 056601. doi:10.1103/PhysRevLett.97.056601

[5] M. Meschke, W. Guichard and J. P. Pekola, "Single-Mode Heat Conduction by Photons," Nature, Vol. 444, 2006, pp. 187-190. doi:10.1038/nature05276

[6] Y. Dubi and M. Di Ventra, "Colloquium: Heat Flow and Thermoelectricity in Atomic and Molecular Junctions," Reviews of Modern Physics, Vol. 83, No. 1, 2011, pp. 131-156.

[7] M. Büttiker, Y. Imry, R. Landauer and S. Pinhas, "Generalized Many-Channel Conductance Formula with Application to Small Rings," Physical Review B, Vol. 31, 1985, pp. 6207-6215. doi:10.1103/PhysRevB.31.6207

[8] L. V. Keldysh, "Diagram Technique for Nonequilibrium Processes," Soviet Physics-JETP, Vol. 20, No. 4, 1965, pp. 1018-1026.

[9] C. Caroli, R. Combescot, P. Nozieres and D. Saint-James, "Direct Calculation of the Tunneling Current," Journal of Physics C: Solid State Physics, Vol. 4, No. 8, 1971, p. 916. doi:10.1088/0022-3719/4/8/018

[10] Y. Meir and N. S. Wingreen, "Landauer Formula for the Current through an Interacting Electron Region," Physical Review Letters, Vol. 68, No. 16, 1992, pp. 2512-2515. doi:10.1103/PhysRevLett.68.2512

[11] A. Ozpineci and S. Ciraci, "Quantum Effects of Thermal Conductance through Atomic Chains," Physical Review B, Vol. 63, No. 12, 2001, Article ID: 125415. doi:10.1103/PhysRevB.63.125415

[12] N. Mingo and L. Yang, "Phonon Transport in Nanowires Coated with an Amorphous Material: An Atomistic Green's Function Approach," Physical Review B, Vol. 68, No. 24, 2003, Article ID: 245406. doi:10.1103/PhysRevB.68.245406

[13] T. Yamamoto and K. Watanabe, 'Nonequilibrium Green's Function Approach to Phonon Transport in Defective Carbon Nanotubes," Physical Review Letters, Vol. 96, No. 25, 2006, Article ID: 255503. doi:10.1103/PhysRevLett.96.255503

[14] K. Yamamoto, H. Ishii, N. Kobayashi and K. Hirose, "Effects of Vacancy Defects on Thermal Conduction of Silicon Nanowire: Nonequilibrium Green's Function Approach," Applied Physics Express, Vol. 4, 2011, Article ID: 085001. doi:10.1143/APEX.4.085001

[15] M. P. Lopez-Sancho, J. M. Lopez-Sancho and J. Rubio, "Quick Iterative Scheme for the Calculation of Transfer Matrices: Application to Mo (100)," Journal of Physics F: Metal Physics, Vol. 14, No. 5, 1984, p. 1205. doi:10.1088/0305-4608/14/5/016

[16] J. Tersoff, "Empirical Interatomic Potential for Silicon with Improved Elastic Properties," Physical Review B, Vol. 38, No. 14, 1988, pp. 9902-9905. doi:10.1103/PhysRevB.38.9902

[17] D. W. Brenner, "Empirical Potential for Hydrocarbons for 
Use in Simulating the Chemical Vapor Deposition of Diamond Films," Physical Review B, Vol. 42, No. 15, 1990, pp. 9458-9471. doi:10.1103/PhysRevB.42.9458
[18] D. Li, Y. Wu, P. Kim, L. Shi, P. Yang and A. Majumdar, "Thermal Conductivity of Individual Silicon Nanowires," Applied Physics Letters, Vol. 83, No. 14, 2003, p. 2934. 